\section{Assessing the value of assertive outreach}

\author{
Qualitative study of process and outcome generation \\ in the UK700 trial
}

TIM WEAVER, PETER TYRER,* JANE RITCHIE and ADRIAN RENTON

\begin{abstract}
Background It is unclear why intensive case management (ICM) failed to reduce hospitalisation in the UK700 trial.
\end{abstract}

\section{Aims To investigate outcome generation in the UK700 trial.}

Method A qualitative investigation was undertaken in one UK700 centre.

Results Both intensive and standard case management practised individual casework, employed assertive outreach with comparable frequency, and performed similarly in the out-patient management of emergencies and inpatient discharge. However, ICM was advantaged in managing some noncompliance and undertaking casework that prevented psychiatric emergencies. Absence of team-based management and bureaucratised access to social care limited the impact of these differences on outcomes and the effective practice of assertive outreach, although this was relevant to only a sub-population of patients.

\section{Conclusions The impact of ICM was} undermined by organisational factors. Sensitive anticipatory casework, which prevents psychiatric emergencies, may make ICM more effective than an exclusive focus on assertive outreach. Our findings demonstrate the value of qualitative research in evaluating complex interventions.

\section{Declaration of interest None.}

Funding detailed in Acknowledgements.

*This paper was accepted before P.T. became Editor of the Journal.
Doubts about the effectiveness of case management for people with severe mental illness were expressed when the care programme approach (CPA) was introduced in the UK (Anonymous, 1995; Marshall, 1996) and evidenced by systematic review (Marshall et al, 2002). The UK700 case management trial assessed whether enhanced outcomes could be achieved under CPA by reducing case-load size. The trial tested the hypothesis that reducing CPA case-loads to $10-15$ patients (intensive case management) would result in less hospitalisation. It was argued that the intensive case management team would be able to practise assertive outreach more extensively because of their reduced caseload and that this would contribute to the hypothesised difference in outcome (Burns et al, 1999). Brief training in assertive outreach was given to the intensive case management team (Burns et al, 1999). However, the findings did not support the hypothesis (Burns et al, 1999), and the impact of assertive outreach on outcomes remains unclear (Tyrer, 2000).

\section{METHOD}

\section{Overview and aims}

We implemented a 2-year qualitative investigation in parallel to the UK700 trial in one centre (St Mary's/St Charles). The aims were to identify the mechanisms by which UK700 trial outcomes were generated and to investigate the nature and impact of forms of assertive casework (with or without outreach) upon the UK700 outcomes.

\section{The UK700 trial}

The UK700 trial has been described elsewhere (Burns et al, 1999; UK700 Group, 1999). In brief, patients with psychosis aged 16-65 years who had at least two prior in-patient admissions were randomly allocated to either standard case management (case-load 1:30-35) or intensive case management (case-load 1:10-15). The trial tested the hypothesis that intensive case management would achieve shorter duration of hospitalisation (primary outcome) over a 2-year follow-up period (UK700 Group, 1999). Assertive outreach and case-loads of 1:10-15 are characteristic of assertive community treatment - an alternative approach to case management with a more secure evidence base (Marshall \& Lockwood, 2002). However, the trial did not evaluate a full-fidelity assertive community treatment intervention (Stein \& Santos, 1998) and did not formally compare assertive and non-assertive case management (Burns et al, 1999).

\section{Qualitative investigation}

The qualitative work had two components. The first was a thematic interview survey of 19 case managers (8 practising standard and 11 practising intensive case management) in post during the 2-year study period starting in December 1995 (Table 1). The second involved longitudinal, patient-based case studies. We investigated case management practice for a sample of patients receiving either intensive or standard case management. Qualitative research conventionally employs purposive sampling to ensure representation of the range and diversity of the study population. However, in the context of the trial we opted for a stratified, quota sampling design with random case selection (Fig. 1). We sampled 40 cases, but 1 intensive management case was lost to follow-up by the UK700 study and also excluded from the case study population. Findings are therefore based on an analysis of 39 cases (19 intensive, 20 standard case management). Owing to staff turnover the median number of case managers allocated to a patient during the study period was 3 (range 1-9) and a total of 77 case manager interviews were completed (42 standard, 35 intensive case management). Interviews were completed with 30 patients (15 standard, 15 intensive case management) and local authority social workers (known as 'care managers') in all 24 cases where they were involved (11 standard, 13 intensive).

\section{Qualitative interviews}

Interviews were based on topic lists drafted after a literature review and refined progressively during fieldwork. Questioning was structured by the interviewer 
Table I Profile of respondents in the investigation of the models of standard and intensive case management

Professional background

Total of staff interviewed Permanent staff Temporary staff

$n$

$n$

Standard team
Community psychiatric nurse
Non-qualified (nursing auxiliary)
Intensive team
Community psychiatric nurse
Occupational therapist
Psychologist
Social worker
Non-qualified $^{3}$

Total

$\begin{array}{lll}6 & 5 & \text { I } \\ 2 & 1 & 1 \\ 4 & 2 & 2 \\ 2 & 1 & 1 \\ 2 & 2 & \\ 1 & 1 \\ 2 & 1 & 1 \\ 19 & 12 & 7\end{array}$

I. Throughout the study period psychologists on the team held posts split between the case management team $(0.5)$ and the local sector team $(0.5)$.

2. Temporary National Health Service appointment of a worker trained in the European Union. Did not have care management responsibilities.

3. Includes psychology graduates and occupational therapist (OT) trainee. Note: two permanent staff (I OT, I nonqualified) started the study period working full-time, but returned to part-time posts after taking maternity leave.

(T.W.) to cover key themes, but was also responsive to issues emerging from respondents' accounts. Thematic interviews with case managers covered case management practice and process, inter-agency working, and the relationship between working context, case management process and primary outcome. Case study interviews investigated similar themes, but also obtained chronological, multi-perspective accounts of each patient's management during the study period. We employed a 'critical incident' approach (Pryce-Jones, 1993) to assess factors in each case influencing the level of success in managing both psychiatric emergencies and duration of admission.

\section{Analysis}

Interviews were tape-recorded, transcribed and downloaded for online coding and analysis using QSR NUD*IST (Scolari/ Sage, 1998). The coding of data operated at three levels. First, each transcript was retained as an individual data source (or case) and coded throughout using trial randomisation variables (case, treatment group, referral source), patient demographics (gender, ethnicity) and service characteristics (number and characteristics of case managers). As with any quantitative database, this coding facilitated analysis within and between the groups - notably between standard and intensive case management cases. Second-level codes were descriptive categories used to label themes identified in sub-sections of a transcript.
These codes related to research questions (e.g. management of psychiatric emergencies, admission, assertive casework) or were 'theoretical', in that they reflected our conceptual approach, which sought to understand outcome generation through investigation of the case management process; for example, assessment, care planning, monitoring and review were coding categories. Third-level codes were either descriptive sub-categories of level-two codes (e.g. assertive casework strategies) or emergent themes identified through the analysis (e.g. mechanisms acting on the frequency and duration of admission). Both of the above examples are described in the findings.

We used this framework to code transcripts online and employed the crossreference, search and text retrieval facilities of NUD*IST to interrogate the data. Second- or third-level codes were applied to 'units' of text of at least one paragraph. This minimised the loss of 'context' when analysing the text yielded by online searches. The analysis of the thematic interviews had the following objectives:

(a) to describe how case managers perceived their roles and practised case management;

(b) to identify formative influences upon case management practice;

(c) to identify differences and similarities between standard and intensive case management.

Analysis of the case studies involved the compilation of case dossiers, comprising multi-perspective accounts of the chronology of each case. These dossiers were analysed to describe casework, and to develop a typology of assertive strategies. We then investigated evidence about the positive or negative impact that key aspects of the context and case management practice (identified through the above analysis) had upon the primary outcome (admission). We compared the effectiveness of standard and intensive case management in preventing or managing psychiatric emergencies and the extent to which admission of patients was avoided or minimised. In all cases evidence of positive or negative impact was only considered where data triangulation provided corroboration from two or more sources (i.e. two or more case managers, care managers and/or the patient; supporting case-note evidence; and/or the reporting of multiple events suggestive of a pattern within the case).

\section{RESULTS}

\section{Thematic interview survey}

\section{Casework roles and practice}

All case managers expressed a consensus that the aims of case management were patient engagement, improved health and social function, and reduced admission. They regarded comprehensive assessment of patient needs and provision of a needsled service as the key mechanisms for achieving these aims. Case managers recognised the usefulness of assertive outreach, but only as a strategy of last resort when preferred 'consensual' management approaches became unsustainable because of significant non-compliance. Case managers felt obliged to address assessed patient need themselves (individual casework). In responding to needs that fell outside their disciplinary training, case managers described two options: to negotiate interventions from other individuals or agencies (brokerage), or to take on clinical casework roles not normally associated with their discipline (genericism). All case managers (both standard and intensive) reported practising individual casework and most adopted both genericism and brokerage at times. However, individual casework coupled with genericism appeared to be the dominant approach.

\section{Formative influences}

There was recognition that being a case manager involved a degree of encroachment 


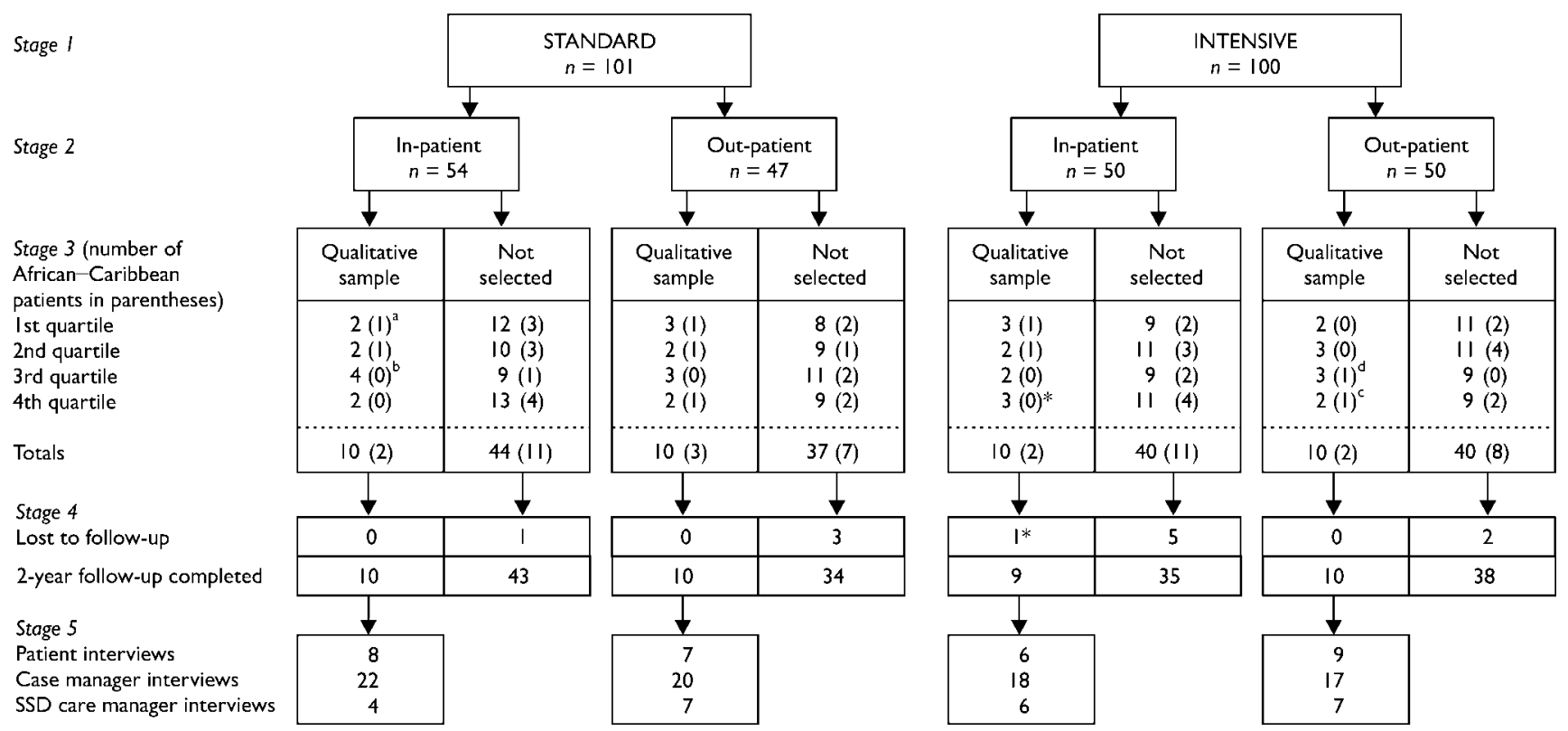

Fig. I Selection of case study sample, case characteristics and extent of available qualitative data. Stage I: patients were stratified by treatment group to which they were randomised in the UK700 trial. Stage 2: each treatment group was stratified by in-patient/out-patient status at randomisation to produce four cells. Stage 3: 10 cases were selected at random from each cell, controlled by minimum-maximum quotas for African-Caribbean patients and phase of recruitment. We ensured proportionate representation of African-Caribbean patients (whose outcomes were subject to secondary hypotheses) and cases randomised at different phases in the recruitment process (owing to the potential for longitudinal changes in case management practice). Case selection was random, but we rejected a case if it meant defined quotas for the above variables were not met in each cell. (Phase in recruitment: 2-4 cases from each quartile divided according to the chronological rank order of randomisation; African-Caribbean: 2-4 cases.) Random case selection satisfied African-Caribbean quotas but substitution by repeated random selection was required in $2 / 40$ cases to meet phase of recruitment quotas (i.e. substitute ${ }^{\text {a }}$ replaced I case selected at ${ }^{b}$, I case at ${ }^{c}$ substituted for a case at ${ }^{d}$ ). Stage 4: Data collection. One intensive case management case $\left(^{*}\right)$ was lost to follow-up and excluded from the outcome group in the UK700 trial analysis. Very limited qualitative data about this case were obtained and it was also dropped from the qualitative case study population. Findings are therefore based on an analysis of 39 cases (19 intensive, 20 standard case management).

Stage 5: completed interviews. The UK700 main outcome analysis used data from 189/20I cases from the St Mary's/St Charles' site.

upon the traditional roles of other professionals. However, the administration and monitoring of medication was not only fundamental to the management of most patients, but also a clinical function that only community psychiatric nurses (CPNs), could legitimately perform. Consequently, CPNs were often called upon to administer medication to patients on the case-loads of non-CPNs and they bore disproportionate responsibility for assertive outreach to patients who were non-compliant with medication. Assertive outreach was seen as difficult and stressful. This undermined teamwork in both standard and intensive case management teams because CPNs perceived limited scope for reciprocal working arrangements.

\section{Comparison of standard and intensive case management practice}

The majority of case managers in both standard and intensive case management interpreted casework as an individual rather than a team responsibility and felt obliged to practise generic casework in order to provide needs-led interventions. Team-based management of patients did not emerge in either the intensive or the standard case management teams. However, the teams reported different expectations about the outcomes they could achieve with patients. The intensive case management team felt a greater pressure of expectation to achieve more in terms of rehabilitation. However, they sometimes reported a tendency to overcommit themselves, difficulty in defining appropriate boundaries to their work and problems setting clinical objectives that matched their potential for intensive casework.

\section{Case studies}

\section{Models of casework}

Individual casework was reported in virtually all case studies, but a generic approach was described in only 16 of 39 cases ( 8 standard, 8 intensive). There were a number of reasons. (a) In approximately half of the sample the patients were compliant and their major social needs were met. Whether under standard or intensive case management, these cases received comparable low-to-medium intensity, non-generic monitoring and usually recorded positive outcomes.

(b) Staff turnover: in some cases, initial management involved a generic approach that was not sustained after reallocation. Sometimes this was because non-recurrent needs were met (e.g. resolving benefit problems). However, case managers who adopted an initial generic approach and addressed the patient's practical and financial needs often enjoyed a dividend in terms of improved engagement. This helped sustain rehabilitative casework and reduced the need for assertive casework. It was difficult for a subsequent case manager to achieve a similar clinical relationship with the patient.

(c) The involvement of care managers and commissioned social care services - 
particularly hostels - limited the role of the case manager.

(d) Non-compliance increased the need for assertive outreach. Case managers reported that assertive outreach undermined patient engagement and the sort of therapeutic alliance required to practise generic casework, particularly at an intensive level.

Internal brokerage was rarely reported (1 standard management case), although external brokerage - mostly through care management - was more common (12 of 39 cases).

\section{Assertive casework}

In nearly half of the case study population $(n=18$; standard case management 8 , intensive 10) patients were reported to be compliant with medication and appointments and not to need any form of assertive casework. Non-compliance by the remaining 21 patients required varying forms of assertive casework.

Patients exhibiting episodic or serial non-compliance with appointments $(n=5$; standard case management 3 , intensive 2 ) were generally compliant with medication, or their compliance was maintained through counselling. In these cases assertive monitoring was practised, but typically involved relatively infrequent scheduled appointments. This did not appear to be difficult for either intensive or standard case management workers to implement.

Episodic or serial non-compliance with medication ( $n=7$; standard case management 4 , intensive 3 ) posed more problems, especially when associated with rapid relapse, increased elusiveness or aggression. In these cases periodic assertive and intensive (i.e. frequent) monitoring was required. An important factor influencing the success of 'episodic assertiveness' was the closeness of monitoring when patients were stable. This could break down in either team owing to staff turnover, but the standard case management team were generally disadvantaged by virtue of their lower frequency of contact. However, experienced standard case management workers who had the ability to identify early signs of relapse and a greater self-confidence about acting independently to initiate management change did accommodate episodic increases in the frequency of contact with individual patients by effective time management and displacement of routine casework. Three cases (2 standard, 1 intensive) were characterised by persistent non-compliance with medication and elusive non-compliance with appointments. These patients had relatively high levels of social functioning, were disinclined to present themselves at services, and were prone to slow, insidious relapse. Even intensive case management workers reported difficulty finding sufficient time to monitor these patients effectively when the responsibility was not shared with colleagues and no other agency had sufficient contact to provide effective monitoring. In all three cases it proved difficult to sustain a consistent assertive approach. Monitoring broke down, patients relapsed and lengthy admissions were recorded (patient A: one admission, 121 days; patient B: three admissions, 133 days; patient C: four admissions, 231 days).

Some patients exhibited persistent noncompliance with medication with nonelusiveness or frequent chaotic presentation at services. The majority of patients who were persistently non-compliant with medication were also non-compliant with appointment systems, but nevertheless tended to present frequently at services permitting close monitoring without outreach $(n=6$; standard case management 3 , intensive 3). These patients were often described as 'chaotic' and required timeintensive, highly assertive casework. Workers using intensive case management were better placed to sustain a high level of contact but they did not perceive this as making a significant impact on primary outcomes. The patients' chaotic nature often generated a need for crisis management and both intensive and standard case management workers could be drawn into very reactive casework. Interventions related to medical compliance and other relapse risk factors such as substance misuse tended to dominate casework and effectively precluded more rehabilitative casework.

\section{Did assertive casework affect the primary outcome?}

For intensive case management to achieve a significant lower mean duration of inpatient stay than standard case management, intensive case managers would need to achieve one, or more, of the following:

(a) enhanced out-patient management, resulting in fewer psychiatric emergencies;

(b) enhanced management of psychiatric emergencies, so that fewer were resolved by patient admission; (c) enhanced discharge planning, which ensured that in-patients were discharged quickly once their symptoms had been managed.

Is there any evidence that enhanced out-patient management resulted in fewer psychiatric emergencies? Figure 2 shows that there was evidence in 7 standard management cases that potential emergencies were averted at least once in the study period (although emergencies did occur in each case at another time). There was just 1 case where no significant emergency was reported during the study period, but this was not attributed to preventive casework. A different pattern was observed among intensive management cases. There were 5 cases in which no significant emergency (and no admission) was reported in the entire study period. In 4 of these cases this was attributed to preventive casework. In a further 7 cases, evidence suggested that emergencies were prevented at certain times although not for the full duration of the study period.

Table 2 shows that proactive casework involving action that was sensitive to individual circumstances and that anticipated crises had particularly positive effects in both samples. This is exemplified by care managers securing or maintaining accommodation (4 standard management cases, 6 intensive). Thorough medical review, involving extended observation of patients, was reported to be critical in 1 standard management case and 3 intensive management cases. The intensive case management team was better able to undertake this work with the thoroughness needed to achieve an enduring preventive effect. The standard case management workers were more likely to be deflected from this casework by other patients who were actively experiencing some form of emergency. The most common explanations for the absence of positive impact were difficult-to-engage, non-compliant and/or elusive patients (3 standard, 6 intensive cases), poor engagement ( 3 standard cases) and discontinuity of management ( 2 standard cases). Some difficult-to-engage intensive case management patients reported non-compliance with appointments because they were intolerant of the more frequent contact attempted by intensive management workers.

Is there any evidence that enhanced management of psychiatric emergencies resulted in fewer being resolved by hospital admission? In 17 cases (8 standard, 9 intensive case management) 

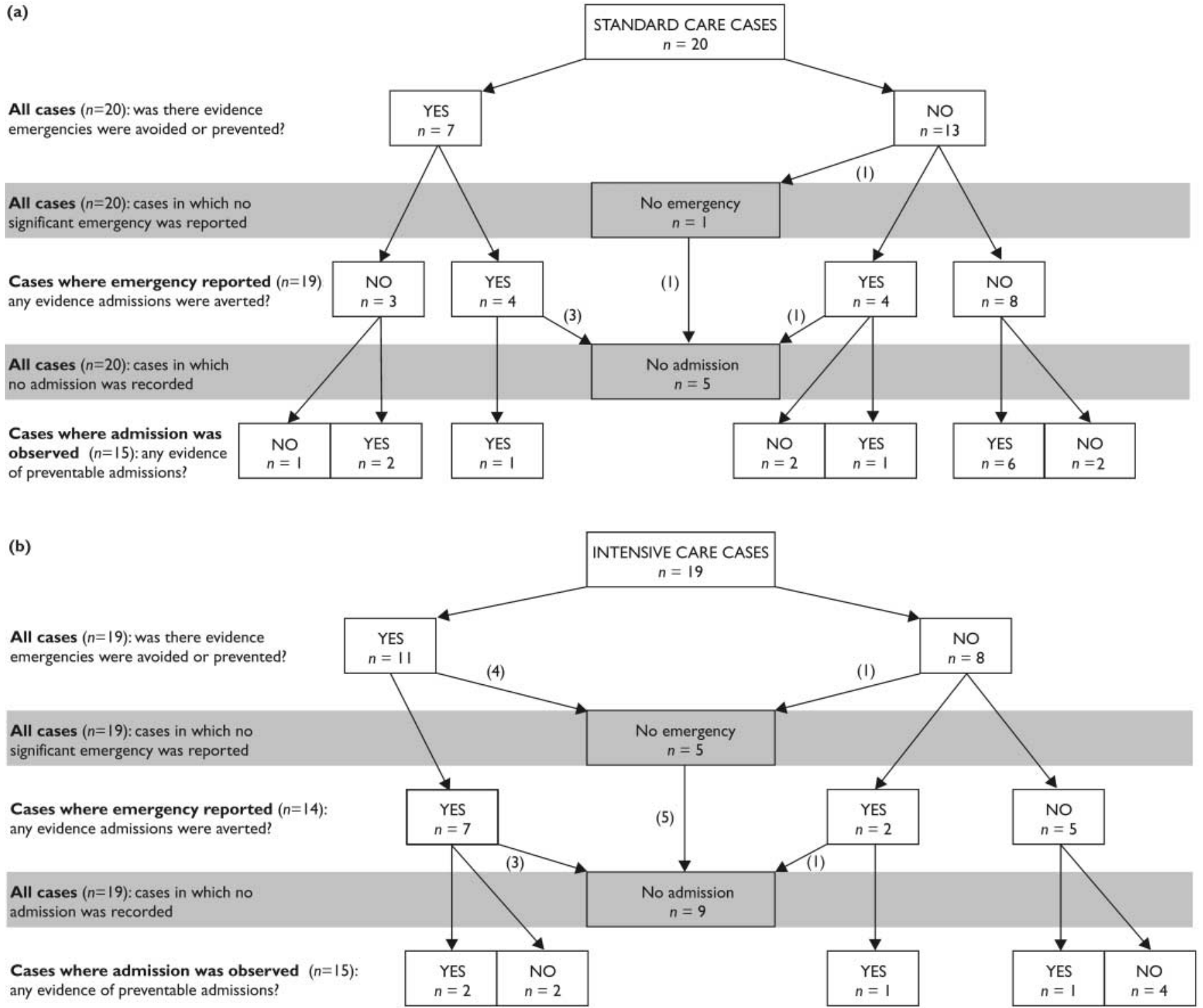

Fig. 2 Comparison in the reported effectiveness of standard case management (a) and intensive case management (b) in preventing hospital admission in a sample of

there was evidence that potential admission had been averted during psychiatric emergencies and in 8 of these $(4$ standard, 4 intensive) this proved sufficient to maintain patients in the community throughout the study period (Fig. 2). Table 2 shows that positive outcomes were most often associated with assertive casework in response to episodic or serial noncompliance (6 standard, 8 intensive). Examples of successful intervention such as rapid medical assessment emphasised the importance of close monitoring and the reactive role of case managers. Complete avoidance of admission throughout the study period (despite recorded psychiatric emergency) was achieved only where informal carers or hostel staff provided a high level of home support (3 standard, 2 intensive management).

Figure 2 shows that potentially preventable admissions occurred in both groups but were more commonly reported among standard management cases (10/15 v. 4/10 intensive management cases). The main factors explaining the failure to avoid preventable admission were a breakdown of close monitoring caused by change of case manager (6 standard, 3 intensive) and delays in securing social care services through care management (2 standard). Non-compliance in response to intensive casework may have been a contributory factor in 2 intensive management cases.
Is there any evidence that enhanced discharge planning ensured that in-patients were discharged quickly once their symptoms had been managed? Among the 25 cases in which admissions were observed, there was evidence of positive impact on the duration of admission in only 1 intensive management case in which a predefined crisis care plan had been developed (Table 2). There were 10 cases in which failures of case management had a negative impact upon the duration of admission (6 standard, 4 intensive). Two factors were identified: first, inappropriate and unsuccessful efforts to manage relapsing patients in the community without admission (2 standard management, 2 intensive management 


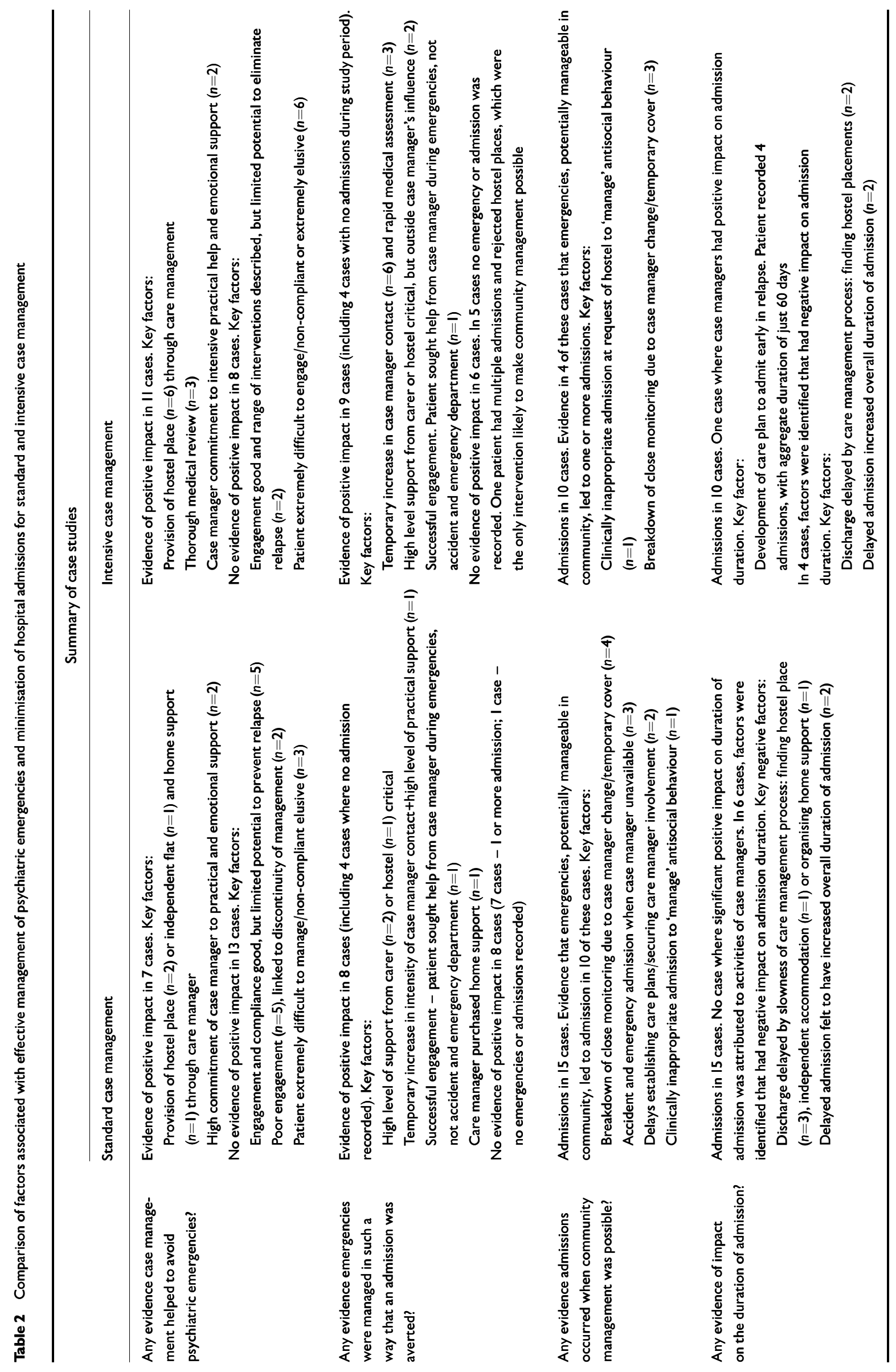


cases), and second, delayed discharge owing to failure to secure hostel or other accommodation (5 standard management, 2 intensive management). There was a strong consensus that the poor coordination and duplication of National Health Service, local authority and hostel assessments extended the duration of some of the longest admissions recorded.

\section{DISCUSSION}

We acknowledge certain limitations of the study. First, despite employing stratified random sampling, as with all qualitative research caution should be exercised in making any inference about the frequency with which the processes and case characteristics we observed were represented within the target population. Although numbers of case studies exhibiting given characteristics have been cited, this is merely to provide illustration of their distribution within the qualitative sample. Second, qualitative data were collected at one of four UK700 sites. We cannot be certain that the processes we observed were present at all sites or that other processes would not have been observed in other sites and non-experimental settings. Notwithstanding these limitations, our findings have major significance for the interpretation of the UK700 findings and service development.

\section{Inter-disciplinary relations}

The trial required workers to define appropriate 'case management' roles and in so doing challenged traditional specialisms. This had an important informative influence on case management practice. In attempting to resolve the resultant interdisciplinary tensions a dominant notion about casework emerged, which was that each case manager practised individual, one-to-one casework, working generically if patient need dictated. As a consequence limited brokerage was practised in either team. The case studies suggested the failure to develop team-based management of patients limited the potential of intensive case management to manage psychiatric emergencies without admission.

\section{Assertive treatment and outreach}

The UK700 study is commendable for monitoring model fidelity, but although this confirmed that intensive case management achieved an increase in patient contact (Burns et al, 2000), it neither explained why this failed to achieve improved outcomes nor assessed the implementation of assertive outreach (Tyrer, 2000).

Given that the promotion of assertive outreach is now UK government policy (Department of Health, 1999), clarity about what actually happened is crucial. Did intensive case management fail because of its lack of adherence to the tenets of assertive outreach? Certainly the training in 'assertive outreach' given to intensive case management teams had limited potential to change practice (Gournay \& Thornicroft, 2000) and its impact was further diluted by staff turnover. Nevertheless, the UK700 study team anticipated that intensive case management teams would be better able to implement assertive outreach by virtue of their reduced case-load (Burns et al, 1999).

Our findings suggest that assertive casework of any sort appeared unnecessary and inappropriate to a substantial proportion of the study population. Although assertive casework was practised, different strategies were observed and the traditional image of 'assertive outreach' with persistently noncompliant and elusive patients was relatively uncommon. Contrary to expectations there was an absence of any marked difference in practice between intensive and standard case management. Both teams managed forms of episodic non-compliance and associated relapse with similar effectiveness, but continuous assertive casework (with outreach) in response to persistent non-compliance appeared unsustainable for both standard and intensive case management because of the absence of teambased management. These factors limited the potential for assertive casework to affect trial outcomes (i.e. to contribute to differences in outcome between standard and intensive case management teams), but we should be cautious about implying that assertive casework was ineffective. Our findings actually suggest that results were mixed.

\section{The usefulness of intensive case management}

The case studies suggested that intensive case management was better able than standard case management to reduce admission by enabling proactive casework involving action that was sensitive to individual circumstances and that anticipated crises. This helped prevent some significant psychiatric emergencies arising. This approach, which we termed sensitive anticipatory action, appeared to have the most significant impact on the frequency and duration of in-patient admission. Sensitive anticipatory action comprises intensive casework (often with non-symptomatic patients) aimed at promoting engagement; undertaking patient-centred medication review; assessing and responding to social care needs; and developing crisis care plans. Ironically, this was the sort of casework that the standard case management team displaced as a 'time management' strategy to practise assertive outreach with noncompliant patients.

\section{Coordination of health and social care}

Our findings support the idea that improved coordination of health and social care is an essential management component and can have significant influence on the frequency and duration of admission. We saw that care plans comprising appropriate accommodation and domiciliary support (arranged through care management) could have preventive potential. However, we also saw how cumbersome arrangements for securing social care through separate care management systems could limit the potential of intensive case management to achieve more rapid discharge of in-patients, resulting in inappropriately lengthy admissions, and that of sensitive anticipatory action to prevent emergencies arising (or to delay their resolution), resulting in a requirement for admission.

\section{Reassessment of the UK700 study findings}

These findings have important policy implications and challenge certain conclusions drawn from the UK700 findings. The UK700 team suggested that more attention needs to be given to ensuring that evidencebased treatment interventions are given to patients rather than focusing on delivery systems (Burns et al, 1999). Clearly, any greater potential of intensive case management will not be realised unless additional contact time can be utilised to deliver effective interventions, but any reduction in our commitment to getting delivery systems right would do community psychiatry a considerable disservice.

Patients with psychotic disorders require a service that responds to changing 
circumstances and provides evidence-based interventions within three distinct phases: sensitive anticipatory action to anticipate emergencies; casework (including assertive casework) during psychiatric emergencies and relapse; and effective planning for speedy discharge. With these goals in mind, we strongly support current moves towards full integration of health and social care within sectorised community mental health teams. We agree with Burns \& Catty (2002) that although the development of such complex interventions presents a challenge to evaluation by randomised controlled trials, we should not fragment services just to enable easier evaluation of individual components. We have shown that implementing concurrent qualitative investigations within such trials of complex interventions enables the treatment process and the interaction of multiple service elements to be investigated (Weaver et al, 1996; Campbell et al, 2000; Crawford et al, 2002).

What, then, of the question of case-load size? Clearly, reducing case-loads is an ineffective intervention when implemented independent of other change. However, this is not evidence that reducing case-loads cannot, or should not, be part of an effective service delivery system. Further analysis of the UK700 study demonstrated as much, for intensive case management did achieve better outcomes in the subpopulation of patients with psychosis and borderline IQ (Tyrer et al, 1999; Hassiotis et al, 2001). This suggests that intensive contact can be useful if applied in appropriate cases. In this respect intensive case management is no different from atypical antipsychotic medication, cognitivebehavioural therapy or even antibiotics. Applied indiscriminately, without consideration for 'dosage', intensive case management may be ineffective or even counterproductive.

Assertive outreach was not practised extensively, and not practised to a significantly greater extent by the intensive case management team. Any advantage enjoyed by intensive case management in practising assertive outreach was undermined by factors independent of case-load size (the absence of team-based management). Assertive outreach could have utility but - like the other evidence-based interventions to which Tyrer (2000) refers - it is unlikely to be deliverable or sustainable in an effective form unless individual, generic casework is abandoned in favour

\section{CLINICAL IMPLICATIONS}

- The potential of assertive casework to affect UK700 trial outcomes was limited. First, in many cases it might not have been appropriate. Second, forms of assertive casework that intensive case managers were better able to sustain appeared to be appropriate in few cases. Third, in most cases where assertiveness was required, both standard and intensive case managers either practised with comparable effectiveness, or were equally restricted by the absence of team-based management.

- Sensitive anticipatory action (SAA) might make intensive case management more effective than an exclusive focus on assertive casework, but enhanced access to social care and team-based management of patients appears to be an important precondition for effective SAA.

- Qualitative research methods that assess the process of outcome generation have a significant part to play in the evaluation of complex interventions.

\section{LIMITATIONS}

- Data were collected at only one of four sites involved in the UK700 trial. We cannot be certain that the processes we observed were present at all sites or that other processes would not have been observed in other centres.

- As in all qualitative research, we cannot estimate the frequency with which the processes and case characteristics we observed were represented within the target population within known confidence limits.

- The qualitative study showed that aspects of the UK700 study context had an unintended formative influence on casework practice. This needs to be acknowledged in assessing the generalisability of findings.

TIM WEAVER, MSc, Department of Social Science and Medicine, Imperial College; PETER TYRER, FRCPsych, Department of Psychological Medicine, Imperial College; JANE RITCHIE, BSc, National Centre for Social Research (formerly SCPR), London, UK; ADRIAN RENTON, FFPHM, Department of Social Science and Medicine, Imperial College, London

Correspondence: Tim Weaver, Department of Social Science and Medicine, Imperial College of Science, Technology and Medicine, Charing Cross Campus, The Reynolds Building, St Dunstan's Road, London W6 8RP,UK. Tel: 0207594 0863; fax: 0207594 0866; e-mail: t.weaver@ic.ac.uk

(First received 22 November 2002, final revision 2 May 2003, accepted 6 May 2003)

of team-based management where casework can be shared when patients' needs so dictate. Assertive casework should be seen as a useful strategy appropriate to an important sub-population of patients, but not as a universal panacea. Tyrer (2000) has previously argued that borrowing key elements of assertive community treatment (an approach designed with the deficiencies of US mental health care in mind) is entirely appropriate given the superior standard of basic psychiatric provision in the UK. To restructure services to accommodate assertive outreach or full-fidelity assertive community treatment teams would be an unhelpful developmental cul-de-sac into which to take UK mental health services.

\section{ACKNOWLEDGEMENTS}

This work was undertaken by the authors, who received funding from the Directorates of Health and Social Care (DHSC) London Research and Development programme. The views expressed in the publication are those of the authors and not necessarily those of the National Health Service or the Department of Health. Tim Weaver has also been supported by a Medical Research Council Health Services Research Fellowship. The authors 
express their gratitude to these funders for supporting this work.

\section{REFERENCES}

Anonymous (1995) Care-management: a disastrous mistake (editorial). Lancet, 345, 399-40I.

Burns, T. \& Catty, J. (2002) Mental health policy and evidence: potentials and pitfalls. Psychiatric Bulletin, 26, 324-327.

_ , Creed, F., Fahy, T., et al (1999) Intensive versus standard case management for severe psychotic illness: a randomised trial. Lancet, 353, 2185-2189.

_, Fiander, M., Kent, A., et al (2000) Effects of caseload size on the process of care of patients with severe psychotic illness: report from the UK700 trial. British Journal of Psychiatry, I77, 427-433.

Campbell, M., Fitzpatrick, R., Haines, A., et al (2000) A framework for the design and evaluation of complex interventions to improve health. BMJ, 32I, 694-696.

Crawford, M., Weaver, T., Rutter, D., et al (2002)

Evaluating new treatments in psychiatry: the potential value of combining qualitative and quantitative research methods. International Review of Psychiatry, 14, 6-11.

Department of Health (1999) A National Service Framework for Mental Health. London: Department of Health.

Gournay, K. \& Thornicroft, G. (2000) Comments of the UK700 case management trial (letter). British Journal of Psychiatry, 177, 371.

Hassiotis, A., Ukoumunne, O. C., Byford, S., et al (200I) Intellectual functioning and outcome of patients with severe psychotic illness randomised to intensive case management: report from the UK700 trial. British Journal of Psychiatry, 178, 166-171.

Marshall, M. (1996) Case management: a dubious practice. BMJ, 312, 523-524.

— \& Lockwood, A. (2002) Assertive community treatment for people with severe mental disorders. Cochrane Library, issue 2. Oxford: Update Software.

\section{_. Gray, A., Lockwood, A., et al (2002) Case}

management for people with severe mental disorders Cochrane Library, issue 2. Oxford: Update Software.

Pryce-Jones, M. (1993) Critical incident techniques as a method of assessing patient satisfaction. In Measurement of Patients' Satisfaction With Their Care (eds R. Fitzpatrick \& A. Hopkins). London: Royal College of Physicians of London.

Scolari/Sage (1998) NUD*IST (Non-numerical Unstructured Data Indexing Searching and Theorizing) Qualitative Data Analysis Program, Version 4. London: Scolari/Sage.

Stein, L. \& Santos, A. (1998) Assertive Community Treatment of Persons with Severe Mental IIIness. New York: WW Norton.

Tyrer, P. (2000) Are small case-loads beautiful in severe mental illness? British Journal of Psychiatry, 177, 386-387.

_, Hassiotis, A., Ukoumunne, O., et al (1999) Intensive case management for psychotic patients with borderline intelligence. Lancet, 354, 999-1000.

UK700 Group (1999) Comparison of intensive and standard case management for patients with psychosis. Rationale of the trial. British Journal of Psychiatry, 174, 74-78.

Weaver, T., Renton, A., Tyrer, P., et al (1996) Combining qualitative data with randomised controlled trials is often useful. BM/, 313, 629 . 